\title{
HUYGENS' PRINCIPLE FOR THE WAVE EQUATION ASSOCIATED WITH THE TRIGONOMETRIC DUNKL-CHEREDNIK OPERATORS
}

\author{
SALEM BEN SAÏD
}

\begin{abstract}
Let $\mathfrak{a}$ be an Euclidean vector space of dimension $N$, and let $k=$ $\left(k_{\alpha}\right)_{\alpha \in \mathscr{R}}$ be a multiplicity function related to a root system $\mathscr{R}$. Let $\Delta(k)$ be the trigonometric Dunkl-Cherednik differential-difference Laplacian. For $(a, t) \in$ $\exp (\mathfrak{a}) \times \mathbb{R}$, denote by $u_{k}(a, t)$ the solution to the wave equation $\Delta(k) u_{k}(a, t)=$ $\partial_{t t} u_{k}(a, t)$, where the initial data are supported inside a ball of radius $R$ about the origin. We prove that $u_{k}$ has support in the shell $\{(a, t) \in \exp (\mathfrak{a}) \times \mathbb{R}|| t \mid-R \leq$ $\|\log a\| \leq|t|+R\}$ if and only if the root system $\mathscr{R}$ is reduced, $k_{\alpha} \in \mathbb{N}$ for all $\alpha \in \mathscr{R}$, and $N$ is odd starting from 3 .
\end{abstract}

\section{Introduction}

It is a well known fact that propagation of waves is different in the two- and in the three-dimensional spaces. For instance, suppose we make a "noise" located near a point $y$ at time $t=0$. This noise can be "heard" at a point $x$ at a later time $t$ only if the distance $x-y$ from $y$ to $x$ is less then $t$. This is true in all dimensions, but the three-dimensional space is of a special interest. After the noise is heard, it moves away and leaves no revibration. This is what is commonly known as Huygens' principle [19]. Mathematically, a second order hyperbolic equation satisfies Huygens' principle if the solution of the corresponding Cauchy problem at some point $x$ depends not on all the Cauchy data, but only on its part on the intersection of the characteristic conoid with vertex $x$ with the Cauchy surface. This means that the fundamental solution of the corresponding Cauchy problem vanishes outside and inside the characteristic conoid, and thus must be located on it.

The problem of classifying all second order hyperbolic differential operators which satisfy the Huygens principle is known as the Hadamard problem [12]. This problem has received a good deal of attention and the literature is extensive $[30,20,8,10,21,31,29,22,14,2,27,3,6,1]$. (Of course, this list of references is not complete.) Nevertheless, this problem is still far from being fully solved. In this paper, we will investigate a natural differential-difference operator of a

Received by the editors October 26, 2004.

2000 Mathematics Subject Classification: 39A70, 43A30, 58G16.

Keywords: Dunkl-Cherednik operators, Huygens' principle, Paley-Wiener theorem, Wave equation. 
similar hyperbolic nature, namely one with the same leading symbol, but with additional reflection terms.

The propagation of waves in $\mathbb{R}^{N}$ is governed by the wave equation

$$
\Delta^{x} u(x, t)=\partial_{t t} u(x, t), \quad \text { for }(x, t) \in \mathbb{R}^{N} \times \mathbb{R} .
$$

Here $\Delta^{x}$ denotes the usual Laplacian operator in the $x$-variable, and the subscript $t$ indicates differentiation in the $t$-variable. It is well known that $(\mathbf{L})$ satisfies Huygens' principle only if $N$ is odd starting from 3 (cf. [8]). In the present work, we will investigate the validity of Huygens' principle for $(\mathbf{L})$ when the Laplacian $\Delta$ is replaced by the so-called trigonometric Dunkl-Cherednik Laplacian operator.

To be more precise, let $W$ be a finite reflection group acting on an Euclidean vector space $(\mathfrak{a},\langle\cdot, \cdot\rangle)$. Put $N:=\operatorname{dim}(\mathfrak{a})$. Let $\mathscr{R}$ be a crystallographic root system, and let $k: \mathscr{R} \rightarrow \mathbb{R}^{+}, \alpha \mapsto k_{\alpha}$ be a multiplicity function satisfying $k_{w \alpha}=k_{\alpha}$ for all $w \in W$. The Dunkl-Cherednik Laplacian operator is given by

$$
\begin{aligned}
\Delta(k)= & \Delta_{A}+\sum_{\alpha \in \mathscr{R}^{+}} k_{\alpha} \operatorname{coth}(\alpha / 2) \partial_{\alpha}+\langle\rho(k), \rho(k)\rangle \\
& +\sum_{\alpha \in \mathscr{R}^{+}} k_{\alpha}\left\{\langle\alpha, \alpha\rangle \frac{e^{-\alpha}}{1-e^{-\alpha}}-4\langle\alpha, \rho(k)\rangle\right\} \triangle_{\alpha}+\sum_{\alpha, \beta \in \mathscr{R}^{+}} k_{\alpha} k_{\beta} \triangle_{\alpha} \triangle_{\beta},
\end{aligned}
$$

where $\Delta_{A}$ denotes the Laplace operator on $A:=\exp (\mathfrak{a}), \rho(k):=\frac{1}{2} \sum_{\alpha \in \mathscr{R}^{+}} k_{\alpha} \alpha$, and $\triangle_{\alpha}:=\left(1-e^{-\alpha}\right)^{-1} \otimes\left(1-r_{\alpha}\right)$, where $r_{\alpha}$ is the reflection in the hyperplane $\langle\alpha\rangle^{\perp}$ orthogonal to the root $\alpha$.

Consider the following Cauchy problem

$$
\Delta(k) u_{k}(a, t)=\partial_{t t} u_{k}(a, t), \quad u_{k}(a, 0)=f(a), \quad \partial_{t} u_{k}(a, 0)=g(a),
$$

where $u_{k}(a, t)$ is a function of $(a, t) \in A \times \mathbb{R}$, and the Cauchy data $f$ and $g$ are two smooth compactly supported functions on $A$. For $R>0$, let $B_{R} \subset \mathfrak{a}$ be the closed ball of radius $R$ about the origin, and let $\mathscr{C}_{R}^{\infty}(A)$ be the space of smooth functions with support contained in $\exp \left(B_{R}\right)$. Using Opdam's Paley-Wiener theorem for the Cherednik transform [25], we prove that $u_{k}(a, t)$ is supported in the shell

$$
\{(a, t) \in A \times \mathbb{R}|| t|-R \leq\|\log a\| \leq| t \mid+R\}
$$

if and only if the root system $\mathscr{R}$ is reduced, $k_{\alpha} \in \mathbb{N}$ for all $\alpha \in \mathscr{R}^{+}$, and $N$ is odd starting from 3 .

As a particular case, if we consider the $W$-invariant situation in the above Cauchy problem, and if the parameter $k$ corresponds to the root multiplicities of a Riemannian symmetric space $G / K$ of the noncompact type, then we recover under the same conditions mentioned above the validity of Huygens' principle for the wave equation on $G / K$ (cf. $[3,14,5]$ ).

Our approach is similar to the one used earlier by Branson, Ólafsson, and Schlichtkrull [3] for the wave equation on Riemannian symmetric spaces of the noncompact type. 


\section{Notations and background}

Let $\mathfrak{a}$ be an Euclidean vector space of dimension $N$ which is equipped with an inner product $\langle\cdot, \cdot\rangle$. For $x \in \mathfrak{a}$, let $\|x\|=\langle x, x\rangle^{1 / 2}$. Let $\mathfrak{a}_{\mathbb{C}}$ be the complexification of $\mathfrak{a}$, and we extend the form $\langle\cdot, \cdot\rangle$ to a bilinear form on $\mathfrak{a}_{\mathbb{C}} \times \mathfrak{a}_{\mathbb{C}}$, again denoted by $\langle\cdot, \cdot\rangle$.

For $\alpha \in \mathfrak{a}^{*}$, denote by $x_{\alpha} \in \mathfrak{a}$ the element corresponding to $\alpha$, and when $\alpha$ is nonzero, we introduce the covector $\breve{\alpha} \in \mathfrak{a}$ of $\alpha$ by the formula $\breve{\alpha}=2 x_{\alpha} /\left\langle x_{\alpha}, x_{\alpha}\right\rangle$. Further, for $\alpha \in \mathfrak{a}^{*} \backslash\{0\}$, let $r_{\alpha}$ be the reflection in the hyperplane $\langle\alpha\rangle^{\perp}$ orthogonal to $\alpha$

$$
r_{\alpha}(\lambda):=\lambda-\lambda(\breve{\alpha}) \alpha \quad \text { for } \lambda \in \mathfrak{a}^{*} .
$$

A crystallographic root system $\mathscr{R}$, possibly non reduced, is a finite subset in $\mathfrak{a}^{*} \backslash\{0\}$, such that: (i) $\mathscr{R}$ spans $\mathfrak{a}^{*}$, (ii) $r_{\alpha}(\mathscr{R})=\mathscr{R}$, and (iii) $\alpha(\breve{\beta}) \in \mathbb{Z}$ for all $\alpha, \beta \in \mathscr{R}$.

The Weyl group $W$ associated with $\mathscr{R}$ is the finite reflection group generated by the reflections $r_{\alpha}$. By duality, $W$ acts on $\mathfrak{a}_{\mathbb{C}}$ and on $A_{\mathbb{C}}:=\exp \left(\mathfrak{a}_{\mathbb{C}}\right)$.

Let $\mathscr{R}^{+} \subset \mathscr{R}$ be a choice of positive roots. A multiplicity function on $\mathscr{R}$ is a $W$-invariant function $k: \mathscr{R} \rightarrow \mathbb{R}$. Setting $k_{\alpha}:=k(\alpha)$, for $\alpha \in \mathscr{R}$, we have $k_{w \alpha}=k_{\alpha}$ for all $w \in W$. Henceforth, $\mathscr{K}$ denotes the set of all multiplicity functions on $\mathscr{R}$, and $\mathscr{K}^{+}$denotes the set of $k=\left(k_{\alpha}\right)_{\alpha \in \mathscr{R}} \in \mathscr{K}$ such that $k_{\alpha} \geq 0$ for all $\alpha \in \mathscr{R}^{+}$.

Set $\breve{\mathscr{R}}:=\{\breve{\alpha} \mid \alpha \in \mathscr{R}\} \subset$ a. Let $\breve{Q}:=\mathbb{Z} \breve{\mathscr{R}}$ be the coroot lattice, and let $P:=(\breve{Q})^{*}$ be the dual of $\breve{Q}$. For $\alpha \in \mathscr{R}$, let $A_{\mathbb{C}}^{\alpha}:=\left\{a \in A_{\mathbb{C}} \mid e^{\alpha}(a)=1\right\}$, and define $A_{\mathbb{C}}^{\text {reg }}$ to be the complementary of $\cup_{\alpha \in \mathscr{R}} A_{\mathbb{C}}^{\alpha}$ in $A_{\mathbb{C}}$. Denote by $\mathbb{C}\left[A_{\mathbb{C}}^{\text {reg }}\right]$ the algebra of regular functions on $A_{\mathbb{C}}^{\text {reg }}$. For $p \in \mathbb{S}\left(\mathfrak{a}_{\mathbb{C}}\right)$, let $\partial(p)$ be the corresponding translation invariant differential operator on $A_{\mathbb{C}}$, so $\partial(p) e^{\mu}=p(\mu) e^{\mu}$, for $\mu \in P$.

Denote by $\mathcal{D}\left[A_{\mathbb{C}}^{\text {reg }}\right]$ the algebra of differential operators on $A_{\mathbb{C}}$ with coefficients in $\mathbb{C}\left[A_{\mathbb{C}}^{\text {reg }}\right]$, and by $\mathcal{D}\left[A_{\mathbb{C}}^{\text {reg }}\right] \otimes \mathbb{C}[W]$ the algebra of differential-reflection operators on $A_{\mathbb{C}}^{\text {reg }}$. For $\xi \in \mathfrak{a}_{\mathbb{C}}$ and $k \in \mathscr{K}$, the Dunkl-Cherednik operators $T(\xi, k) \in$ $\mathcal{D}\left[A_{\mathbb{C}}^{\mathrm{reg}}\right] \otimes \mathbb{C}[W]$ are defined by

$$
T(\xi, k):=\partial(\xi)-\rho(k)(\xi)+\sum_{\alpha \in \mathscr{R}^{+}} k_{\alpha} \alpha(\xi) \frac{1}{1-e^{-\alpha}}\left(1-r_{\alpha}\right),
$$

where

$$
\rho(k):=\frac{1}{2} \sum_{\alpha \in \mathscr{R}^{+}} k_{\alpha} \alpha \in \mathfrak{a}_{\mathbb{C}^{*}}^{*}
$$

In particular, for any $\xi, \eta \in \mathfrak{a}_{\mathbb{C}}$, we have $T(\xi, k) T(\eta, k)=T(\eta, k) T(\xi, k)$. We refer to $[7,25,18]$ for more details on the Dunkl-Cherednik operators.

Let $f$ and $g$ be two elements in the space $\mathscr{C}_{c}^{\infty}(A)$ of smooth compactly supported functions on $A$, and set

$$
\langle f, g\rangle_{k}:=\int_{A} f(a) \overline{g(a)} d \mu(a)
$$


where

$$
d \mu(a):=\prod_{\alpha \in \mathscr{R}^{+}}\left|a^{\alpha / 2}-a^{-\alpha / 2}\right|^{2 k_{\alpha}} d a,
$$

and $d a$ is the Lebesgue measure on $A$ normalized by $\operatorname{Vol}(A / \exp (\breve{Q}))=1$. By [25, Lemma 7.8]

$$
\langle T(\xi, k) f, g\rangle_{k}=\left\langle f,-w_{0} T\left(w_{0}(\bar{\xi}), k\right) w_{0} g\right\rangle_{k},
$$

where $w_{0}$ is the longest element in $W$. Fix an orthonormal basis $\left\{\xi_{i}\right\}_{i=1}^{N}$ on $\mathfrak{a}$, and set $\triangle_{\alpha}:=\left(1-e^{-\alpha}\right)^{-1} \otimes\left(1-r_{\alpha}\right)$. Then

$$
\begin{aligned}
\Delta(k):= & \sum_{i=1}^{N} T\left(\xi_{i}, k\right)^{2} \\
= & \sum_{i=1}^{N} \partial\left(\xi_{i}\right)^{2}+\sum_{\alpha \in \mathscr{R}^{+}} k_{\alpha} \operatorname{coth}(\alpha / 2) \partial_{\alpha}+\langle\rho(k), \rho(k)\rangle \\
& +\sum_{\alpha \in \mathscr{R}^{+}} k_{\alpha}\left\{\langle\alpha, \alpha\rangle \frac{e^{-\alpha}}{1-e^{-\alpha}}-4\langle\alpha, \rho(k)\rangle\right\} \triangle_{\alpha}+\sum_{\alpha, \beta \in \mathscr{R}^{+}} k_{\alpha} k_{\beta} \triangle_{\alpha} \triangle_{\beta} .
\end{aligned}
$$

This formula can be obtained by following [18], where a similar statement was proved for the Heckman-Cherednik operators.

A crucial key in Heckman-Opdam's theory of hypergeometric functions, is the existence of eigenfunctions for the Dunkl-Cherednik system of differential equations.

Theorem 1. (cf. [25]) If $k \in \mathscr{K}^{+}$, then there exists an open subset $U$ of $\exp (i \mathfrak{a})$ containing the identity element e such that there exists a holomorphic function $\mathbb{G}$ on $\mathfrak{a}_{\mathbb{C}}^{*} \times \mathscr{K}^{+} \times A U$ with the following properties

$$
\begin{aligned}
& T^{a}(\xi, k) \mathbb{G}(\lambda, k, a)=\lambda(\xi) \mathbb{G}(\lambda, k, a), \\
& \mathbb{G}(\lambda, k, \mathbf{e})=1 .
\end{aligned}
$$

Here the superscript denotes the relevant variable. Further, for $a \in A$ and $\lambda \in \mathfrak{a}_{\mathbb{C}}^{*}$,

$$
|\mathbb{G}(\lambda, k, a)| \leq|W|^{1 / 2} e^{\|\log a\|\|\operatorname{Re}(\lambda)\|} .
$$

Let $f \in \mathscr{C}_{c}^{\infty}(A)$ and $\lambda \in \mathfrak{a}^{*}$. Following [26], we define the Cherednik transform of $f$ by

$$
\mathscr{F}_{k}(f)(\lambda)=\int_{A} f(a) \mathbb{G}\left(-i w_{0} \lambda, k, w_{0} a\right) d \mu(a),
$$

where $d \mu(a)$ is given by (2.2). Using (2.3), one can check that

$$
\mathscr{F}_{k}(T(\xi, k) f)(\lambda)=-i \lambda(\xi) \mathscr{F}_{k}(f)(\lambda) .
$$

In particular, $\mathscr{F}_{k}(\Delta(k) f)(\lambda)=-\|\lambda\|^{2} \mathscr{F}_{k}(f)(\lambda)$. 
By [25, Theorem 9.13], the inverse transform of $\mathscr{F}_{k}$ is given by

$$
f(a)=\int_{\mathfrak{a}^{*}} \mathscr{F}_{k}(f)(\lambda) \mathbb{G}(i \lambda, k, a) \prod_{\alpha \in \mathscr{R}_{+}^{0}}\left(1-\frac{k_{\alpha}^{0}}{i \lambda(\breve{\alpha})}\right) d \nu(\lambda) .
$$

Here $\mathscr{R}_{+}^{0}:=\mathscr{R}^{0} \cap \mathscr{R}^{+}$, where $\mathscr{R}^{0}$ denotes the set of un-multiplicable roots in $\mathscr{R}$, $k_{\alpha}^{0}:=\frac{1}{2} k_{\alpha / 2}+k_{\alpha}$ for $\alpha \in \mathscr{R}_{+}^{0}$, and

$$
d \nu(\lambda):=\frac{(2 \pi)^{-N}|W|^{-2} \widetilde{c}(\rho(k), k)^{2}}{\widetilde{c}(i \lambda, k) \widetilde{c}(-i \lambda, k)} d \lambda,
$$

where $\widetilde{c}$ is the meromorphic function on $\mathfrak{a}_{\mathbb{C}}^{*} \otimes \mathscr{K}$ defined by

$$
\widetilde{c}(\lambda, k)=\prod_{\alpha \in \mathscr{R}^{+}} \frac{\Gamma\left(\lambda(\breve{\alpha})+\frac{1}{2} k_{\alpha / 2}\right)}{\Gamma\left(\lambda(\breve{\alpha})+\frac{1}{2} k_{\alpha / 2}+k_{\alpha}\right)} .
$$

In the sequel, Opdam's Paley-Wiener theorem for $\mathscr{F}_{k}$ is needed. Let $B_{R}$ be the closed ball in $\mathfrak{a}$ with radius $R>0$ about the origin. Denote by $\mathscr{C}_{R}^{\infty}(A)$ the space of smooth functions on $A$ with support contained in $\exp \left(B_{R}\right)$. Let $\mathscr{H}_{R}\left(\mathfrak{a}_{\mathbb{C}}^{*}\right)$ be the space of entire functions $f$ on $\mathfrak{a}_{\mathbb{C}}^{*}$ with the property that for each integer $M>0$, there exists a constant $\alpha_{M}$ such that

$$
|f(\lambda)| \leq \alpha_{M}(1+\|\lambda\|)^{-M} e^{R\|\operatorname{Im}(\lambda)\|}, \quad \text { for all } \lambda \in \mathfrak{a}_{\mathbb{C}}^{*}
$$

The following is a weaker version of [25, Theorem 8.6].

Theorem 2. If $k \in \mathscr{K}^{+}$, then the Cherednik transform $\mathscr{F}_{k}$ maps $\mathscr{C}_{R}^{\infty}(A)$ bijectively onto $\mathscr{H}_{R}\left(\mathfrak{a}_{\mathbb{C}}^{*}\right)$.

\section{The wave equation for the Dunkl-Cherednik operators}

For $k \in \mathscr{K}^{+}$, consider the following Cauchy problem for the wave equation associated with the Dunkl-Cherednik Laplacian operators

$$
\begin{gathered}
\Delta(k) u_{k}(a, t)=\partial_{t t} u_{k}(a, t), \quad(a, t) \in A \times \mathbb{R} \\
u_{k}(a, 0)=f(a), \quad \partial_{t} u_{k}(a, 0)=g(a) .
\end{gathered}
$$

Here we assume that the functions $f$ and $g$ belong to the space $\mathscr{C}_{R}^{\infty}(A)$. By the principle of finite propagation speed (which holds for almost every wave equation $[9]), u_{k}(\cdot, t)$ is compactly supported for every fixed $t \in \mathbb{R}$. For the time being, we assume that $k \in \mathscr{K}^{+}$and $N \geq 1$.

Set $U_{k}(a, t):=\left[\begin{array}{c}u_{k}(a, t) \\ \partial_{t} u_{k}(a, t)\end{array}\right]$. Thus, we may rewrite the wave equation in

$$
\partial_{t} U_{k}(a, t)=\left[\begin{array}{cc}
0 & 1 \\
\Delta(k) & 0
\end{array}\right] U_{k}(a, t)
$$


Applying the Cherednik transform $\mathscr{F}_{k}$ to both sides above, and using the fact that $\mathscr{F}_{k}(\Delta(k) f)(\lambda)=-\|\lambda\|^{2} \mathscr{F}_{k}(f)(\lambda)$, we obtain

$$
\partial_{t} \mathscr{F}_{k}\left(U_{k}(\cdot, t)\right)(\lambda)=\left[\begin{array}{cc}
0 & 1 \\
-\|\lambda\|^{2} & 0
\end{array}\right] \mathscr{F}_{k}\left(U_{k}(\cdot, t)\right)(\lambda):=\mathbb{A}_{k}\left(U_{k}(\cdot, t)\right)(\lambda) .
$$

Solving this ordinary differential equation, we get

$$
\mathscr{F}_{k}\left(U_{k}(\cdot, t)\right)=e^{t \mathbb{A}} \mathscr{F}_{k}\left(U_{k}(\cdot, 0)\right)(\lambda),
$$

where

That is*

$$
e^{t \mathbb{A}}=\left[\begin{array}{cc}
\cos (t\|\lambda\|) & \sin (t\|\lambda\|) /\|\lambda\| \\
-\|\lambda\| \sin (t\|\lambda\|) & \cos (t\|\lambda\|)
\end{array}\right]
$$

$$
\mathscr{F}_{k}\left(u_{k}(\cdot, t)\right)(\lambda)=\mathscr{F}_{k}(f)(\lambda) \cos (t\|\lambda\|)+\mathscr{F}_{k}(g)(\lambda) \frac{\sin (t\|\lambda\|)}{\|\lambda\|} .
$$

Begin with a solution $u_{k}(a, t)$ to the Cauchy problem (3.1), with Cauchy data $(f, g)$. Therefore, $u_{k}(a,-t)$ solves $(3.1)$ with initial data $(f,-g)$. Thus, the time is reversible, except for a minus sign that may appear when the second Cauchy datum $g$ or its Cherednik transform are involved. So the past is determined by the present as well as the future. We shall present arguments valid for $t>0$, and formulate the suitably altered statements for $t \in \mathbb{R}$ without further proof.

By the inversion formula of $\mathscr{F}_{k}$, we have

$$
\begin{gathered}
u_{k}(a, t)=(2 \pi)^{-N}|W|^{-2} \int_{\mathfrak{a}^{*}}\left\{\mathscr{F}_{k}(f)(\lambda) \cos (t\|\lambda\|)+\mathscr{F}_{k}(g)(\lambda) \frac{\sin (t\|\lambda\|)}{\|\lambda\|}\right\} \times \\
\mathbb{G}(i \lambda, k, a) \prod_{\alpha \in \mathscr{R}_{+}^{0}}\left(1-\frac{k_{\alpha}^{0}}{i \lambda(\breve{\alpha})}\right) \frac{d \lambda}{|c(\lambda, k)|^{2}}
\end{gathered}
$$

where

$$
c(\lambda, k):=\frac{\widetilde{c}(i \lambda, k)}{\widetilde{c}(\rho(k), k)} .
$$

Using the spherical coordinates, we obtain

$$
\begin{array}{r}
u_{k}(a, t)=(2 \pi)^{-N}|W|^{-2} \int_{0}^{\infty} \int_{S}\left\{\mathscr{F}_{k}(f)(r \omega) \cos (t r)+\mathscr{F}_{k}(g)(r \omega) \frac{\sin (t r)}{r}\right\} \times \\
(3.2) \quad \mathbb{G}(i r \omega, k, a) \prod_{\alpha \in \mathscr{R}_{+}^{0}}\left(1-\frac{k_{\alpha}^{0}}{i r \omega(\breve{\alpha})}\right) \frac{d \omega}{|c(r \omega, k)|^{2}} r^{N-1} d r,
\end{array}
$$

where $S \ni \omega$ is the unit sphere in $\mathfrak{a}^{*}$, and $d \omega$ is the $O(\mathfrak{a})$-invariant measure on $S$.

${ }^{*}$ Using the Plancherel formula for the transform $\mathscr{F}_{k}[25$, Theorem 3.19], we can show that the total energy of the solution $u_{k}$ is a conserved quantity, i.e. the total energy of $u_{k}$ is independent of the variable $t$ : As a consequence, we deduce that the solutions to the wave equation are uniquely determined by the initial Cauchy data. 
On the other hand, by means of $\Gamma(2 z)=\pi^{-1 / 2} 2^{2 z-1} \Gamma(z) \Gamma\left(z+\frac{1}{2}\right)$, we may rewrite (2.6) as

$$
\begin{aligned}
\widetilde{c}(\lambda, k) & =\prod_{\alpha \in \mathscr{R}_{+}^{0}} \frac{2^{-k_{\alpha / 2}} \Gamma(\lambda(\breve{\alpha})) \Gamma\left(\lambda(\breve{\alpha})+\frac{1}{2}\right)}{\Gamma\left(\lambda(\breve{\alpha})+\frac{1}{2}\left(k_{\alpha / 2}+1\right)\right) \Gamma\left(\lambda(\breve{\alpha})+k_{\alpha}^{0}\right)} \\
& =\prod_{\alpha \in \mathscr{R}_{+}^{0}} \lambda(\breve{\alpha})^{-1} \prod_{\alpha \in \mathscr{R}_{+}^{0}} \frac{2^{-k_{\alpha / 2}} \Gamma(\lambda(\breve{\alpha})+1) \Gamma\left(\lambda(\breve{\alpha})+\frac{1}{2}\right)}{\left.\Gamma\left(\lambda^{\prime}\right)+\frac{1}{2}\left(k_{\alpha / 2}+1\right)\right) \Gamma\left(\lambda(\breve{\alpha})+k_{\alpha}^{0}\right)} .
\end{aligned}
$$

Thus $|c(\lambda, k)|^{-2}=\mathscr{P}(\lambda, k) \eta(\lambda, k)$, where $\lambda \mapsto \mathscr{P}(\lambda, k)$ is a homogeneous holomorphic polynomial of degree $2\left|\mathscr{R}_{+}^{0}\right|$, and $\lambda \mapsto \eta(\lambda, k)$ is a meromorphic function with the same poles as $|c(\lambda, k)|^{-2}$, such that $\eta(0, k) \neq 0$. Notice that, when the root system $\mathscr{R}$ is reduced, we have

$$
\widetilde{c}(\lambda, k)=\prod_{\alpha \in \mathscr{R}^{+}} \frac{\Gamma(\lambda(\breve{\alpha}))}{\Gamma\left(\lambda(\breve{\alpha})+k_{\alpha}\right)},
$$

and if in addition $k_{\alpha} \in \mathbb{N}$, then we obtain

$$
\widetilde{c}(\lambda, k)=\prod_{\alpha \in \mathscr{R}+} \prod_{m=0}^{k_{\alpha}-1}(\lambda(\breve{\alpha})+m)^{-1},
$$

and

$$
\begin{aligned}
|c(\lambda, k)|^{-2} & =c(\rho(k), k)^{2} \prod_{\alpha \in \mathscr{R}^{+}} \lambda(\breve{\alpha})^{2} \prod_{\alpha \in \mathscr{R}^{+}} \prod_{m=1}^{k_{\alpha}-1}\left(\lambda(\breve{\alpha})^{2}+m^{2}\right) \\
& =\mathscr{P}(\lambda, k) \eta(\lambda, k),
\end{aligned}
$$

where now $\eta$ is a holomorphic function. Thus, in general, (3.2) can be written as

$$
u_{k}(a, t)=(2 \pi)^{-N}|W|^{-2} \int_{0}^{\infty}\left\{\Phi_{k}(r, a) \cos (t r)+\Psi_{k}(r, a) \frac{\sin (t r)}{r}\right\} d r
$$

where

$\Phi_{k}(r, a):=r^{N-1} \int_{S} \mathscr{F}_{k}(f)(r \omega) \mathbb{G}(i r \omega, k, a) \prod_{\alpha \in \mathscr{R}_{+}^{0}}\left(1-\frac{k_{\alpha}^{0}}{i r \omega(\breve{\alpha})}\right) \mathscr{P}(r \omega, k) \eta(r \omega, k) d \omega$,

and

$\Psi_{k}(r, a):=r^{N-1} \int_{S} \mathscr{F}_{k}(g)(r \omega) \mathbb{G}(i r \omega, k, a) \prod_{\alpha \in \mathscr{R}_{+}^{0}}\left(1-\frac{k_{\alpha}^{0}}{i r \omega(\breve{\alpha})}\right) \mathscr{P}(r \omega, k) \eta(r \omega, k) d \omega$.

Let $\widetilde{\mathbb{S}} \subset \mathfrak{a}_{\mathbb{C}}^{*}$ be the singular set of $\eta(\cdot, k)$, and let $\mathbb{S}=\{\|\lambda\| \mid \lambda \in \widetilde{\mathbb{S}}\}$. If $c^{-1}(\cdot, k)$ is entire, then $\mathbb{S}=\emptyset$. Otherwise $\mathbb{S}$ attains a minimum $\gamma_{0} \neq 0$. If $\|\lambda\| \in \mathbb{S}_{0}:=$ $\left\{\|\lambda\| \in \mathbb{S} \mid\|\lambda\|=\gamma_{0}\right\}$, then

$$
\lambda= \pm i \min \left(\frac{1}{2}\left(k_{\alpha / 2}+1\right), k_{\alpha}^{0}\right) \frac{\alpha}{2},
$$


for some $\alpha \in \mathscr{R}_{+}^{0}$, and $\gamma_{0}=\min \left(\frac{1}{2}\left(k_{\alpha / 2}+1\right), k_{\alpha}^{0}\right) \frac{|\alpha|}{2}>0$. (We exclude the case where $k_{\alpha}=k_{\alpha / 2}=0$ for all $\alpha \in \mathscr{R}_{+}^{0}$, since this case corresponds to the classical setting where $\widetilde{c} \equiv 1$.) Recall that when $\mathscr{R}$ is reduced and $k_{\alpha} \in \mathbb{N}$, the function $\lambda \mapsto \eta(\lambda, k)$ is entire. We refer to this case by writing $\gamma_{0}=\infty$.

For $(z, \omega) \in \mathbb{C} \times S$, denote by $\eta(z, \omega, k):=\eta(z \omega, k)$ the meromorphic function in the variable $z \in \mathbb{C}$, for fixed $\omega \in S$. Here $\eta(z \omega, k)$ is the function obtained from $\eta(r \omega, k)$ by replacing $r$ by $z$.

From the above discussion it follows that, for fixed $\omega \in S$, the singularities of $\eta(z, \omega, k)$ are poles, and

$$
\left.\left.\gamma_{0}=\min \{|z| \mid \eta(\cdot, \omega, k) \text { has poles at } z \text { for some } \omega \in S\} \in\right] 0, \infty\right],
$$

(recall that $\gamma_{0}=\infty$ if and only if $\mathscr{R}$ is reduced and $k_{\alpha} \in \mathbb{N}$ for all $\alpha$ ).

For $\gamma>0$, set

$$
L(\gamma):=\{\tau+i \sigma \mid-\gamma<\sigma<\gamma\} .
$$

Observe that $\eta(\cdot, \omega, k)$ is holomorphic on $L\left(\gamma_{0}\right)$, for each $\omega \in S$. As the measure $d \omega$ on $S$ is $O(\mathfrak{a})$-invariant, we have:

Lemma 3. If $N-1$ is even, then for fixed $a \in A$, the integral formulas for $\Phi_{k}$ and $\Psi_{k}$ continue analytically to even functions for $r \in L\left(\gamma_{0}\right)$.

Henceforth, we will assume that $N-1$ is even. So we can rewrite (3.3) as

$$
u_{k}(a, t)=\frac{1}{2}(2 \pi)^{-N}|W|^{-2} \int_{\mathbb{R}}\left\{\Phi_{k}(r, a)+\frac{\Psi_{k}(r, a)}{i r}\right\} e^{i r t} d r .
$$

By [3, Equation (9)], if $\gamma<\gamma_{0} \neq \infty$, there exists a constant $c_{k}$ and $M \in \mathbb{N}$, both independent of $\gamma$, such that

$$
|\eta(z, \omega, k)| \leq c_{k}\left(\gamma_{0}-\gamma\right)^{-1}(1+|z|)^{M}, \quad \text { for all } z \in \overline{L(\gamma)} \text { and } \omega \in S .
$$

Clearly, if $\gamma_{0}=\infty$, i.e. $\mathscr{R}$ is reduced and $k_{\alpha} \in \mathbb{N}$ for all $\alpha$, then

$$
|\eta(z, \omega, k)| \leq c_{k}(1+|z|)^{M}, \quad \text { for all } z \in \mathbb{C} \text { and } \omega \in S .
$$

Now let $z \in \overline{L(\gamma)}$. In view of Lemma 3 , and since $S$ is compact, we have

$$
\begin{aligned}
& \left|\Phi_{k}(z, a)\right| \\
& \leq|z|^{N-1} \int_{S}\left|\mathscr{F}_{k}(f)(z \omega)\right||\mathbb{G}(i z \omega, k, a)| \prod_{\alpha \in \mathscr{R}_{+}^{0}}\left|1-\frac{k_{\alpha}^{0}}{i z \omega(\breve{\alpha})}\right||\mathscr{P}(z \omega, k)||\eta(z, \omega, k)| d \omega \\
& \leq c_{k}^{\prime}|z|^{N-1+\left|\mathscr{R}_{+}^{0}\right|} \sup _{\omega \in S}\left\{\left|\mathscr{F}_{k}(f)(z \omega)\right||\mathbb{G}(i z \omega, k, a)| \prod_{\alpha \in \mathscr{R}_{+}^{0}}\left|i z \omega(\breve{\alpha})-k_{\alpha}^{0}\right||\eta(z, \omega, k)|\right\},
\end{aligned}
$$

and

$$
\begin{aligned}
& \left|\frac{\Psi_{k}(z, a)}{z}\right| \\
& \leq c_{k}^{\prime}|z|^{N-2+\left|\mathscr{R}_{+}^{0}\right|} \sup _{\omega \in S}\left\{\left|\mathscr{F}_{k}(g)(z \omega)\right||\mathbb{G}(i z \omega, k, a)| \prod_{\alpha \in \mathscr{R}_{+}^{0}}\left|i z \omega(\breve{\alpha})-k_{\alpha}^{0}\right||\eta(z, \omega, k)|\right\} .
\end{aligned}
$$


If $z=0$, the last estimate gives a problem if $N=1$ and $\left|\mathscr{R}_{+}^{0}\right|=0$. Henceforth, we add the assumption $N>1$ if $\left|\mathscr{R}_{+}^{0}\right|=0$. In view of the Paley-Wiener Theorem 2 applied to the Cauchy data $(f, g)$, and in the light of (2.4) and (3.5), we conclude that for any $M \in \mathbb{N}$ there exist two constants $\alpha_{M}(k)$ and $\beta_{M}(k)$, depending only on $(f, g), N$ and $k$ but not on $z$, such that

$$
\left|\Phi_{k}(z, a)\right| \leq \alpha_{M}(k)\left(\gamma_{0}-\gamma\right)^{-1}(1+|z|)^{-M} e^{|\operatorname{Im}(z)|[R+\|\log a\|]},
$$

and

$$
\left|\frac{\Psi_{k}(z, a)}{z}\right| \leq \beta_{M}(k)\left(\gamma_{0}-\gamma\right)^{-1}(1+|z|)^{-M} e^{|\operatorname{Im}(z)|[R+\|\log a\|]},
$$

for $z \in \overline{L(\gamma)}$ and $\gamma<\gamma_{0} \neq \infty$. If $\gamma_{0}=\infty$, the above two inequalities hold without the term $\left(\gamma_{0}-\gamma\right)^{-1}$.

Next, we will shift the contour in the integral (3.4) from $\mathbb{R}$ to $\mathbb{R}+i \gamma$, so that, by means of the above inequalities, we have

$$
\begin{aligned}
\left|u_{k}(a, t)\right| & =\frac{1}{2}(2 \pi)^{-N}|W|^{-2}\left|\int_{\mathbb{R}}\left\{\Phi_{k}(z, a)+\frac{\Psi_{k}(z, a)}{i z}\right\} e^{i z t} d z\right| \\
& =\frac{1}{2}(2 \pi)^{-N}|W|^{-2} e^{-\gamma t}\left|\int_{\mathbb{R}}\left\{\Phi_{k}(\tau+i \gamma, a)+\frac{\Psi_{k}(\tau+i \gamma, a)}{i(\tau+i \gamma)}\right\} e^{i t \tau} d \tau\right| \\
& \leq \begin{cases}c_{M}(k)\left(\gamma_{0}-\gamma\right)^{-1} e^{-\gamma(t-R-\|\log a\|)} \int_{\mathbb{R}}(1+|\tau|)^{-M} d \tau & \text { if } \gamma<\gamma_{0} \neq \infty \\
c_{M}(k) e^{-\gamma(t-R-\|\log a\|)} \int_{\mathbb{R}}(1+|\tau|)^{-M} d \tau & \text { if } \gamma<\gamma_{0}=\infty\end{cases}
\end{aligned}
$$

The following theorem collects all of the above computations and discussions.

Theorem 4. Assume that $N-1$ is even, with the extra condition $N>1$ if $\left|\mathscr{R}_{+}^{0}\right|=0$. Let $u_{k}$ be a solution to the Cauchy problem (3.1) where the Cauchy data $(f, g)$ belong to $\mathscr{C}_{R}^{\infty}(A)$. Denote by $\gamma_{0} \neq 0$ the minimal distance from a singular point of $|c(\lambda, k)|^{-2}$ to the origin in $\mathfrak{a}_{\mathbb{C}}^{*}$, and suppose that $0<\gamma<\gamma_{0}$.

(i) If $\gamma_{0} \lesseqgtr \infty$, then there exists a constant $c(k)$ depending only on $N, k$ and $(f, g)$, such that

$$
\left|u_{k}(a, t)\right| \leq c(k)\left(\gamma_{0}-\gamma\right)^{-1} e^{-\gamma(|t|-R-\|\log a\|)}, \quad \forall(a, t) \in A \times \mathbb{R} .
$$

(ii) If $\gamma_{0}=\infty$, then

$$
\left|u_{k}(a, t)\right| \leq c(k) e^{-\gamma(|t|-R-\|\log a\|)}, \quad \forall(a, t) \in A \times \mathbb{R} .
$$

Before we state the main result of the paper, let us make the following observation regarding the support of $u_{k}(a, t)$ : Recall that

$$
\mathscr{F}_{k}\left(u_{k}(\cdot, t)\right)(\lambda)=\mathscr{F}_{k}(f)(\lambda) \cos (t\|\lambda\|)+\mathscr{F}_{k}(g)(\lambda) \frac{\sin (t\|\lambda\|)}{\|\lambda\|},
$$

where $f$ and $g$ belong to $\mathscr{C}_{R}^{\infty}(A)$. Observe that $\cos (t\|\lambda\|)$ and $\sin (t\|\lambda\|) /\|\lambda\|$ can be extended to entire functions on $\mathfrak{a}_{\mathbb{C}}^{*}$. Indeed, for $z \in \mathbb{C}$, the functions $\cos z$ and $\sin z / z$ are both even, and thus we may consider the functions $\cos (\sqrt{z})$ and $\sin (\sqrt{z}) / \sqrt{z}$ which are entire analytic functions of $z$ (even though $\sqrt{z}$ it is not 
single-valued). Thus, the analytic extensions of $\cos (t\|\lambda\|)$ and $\sin (t\|\lambda\|) /\|\lambda\|$, respectively, are

$$
\cos \left(t\langle\lambda, \lambda\rangle^{1 / 2}\right), \quad \frac{\sin \left(t\langle\lambda, \lambda\rangle^{1 / 2}\right)}{\langle\lambda, \lambda\rangle^{1 / 2}} .
$$

Further, if we write $\langle\lambda, \lambda\rangle^{1 / 2}=u+i v$ and use the fact that $|\cos (u+i v)|$ and $\left|\frac{\sin (u+i v)}{u+i v}\right|$ are both bounded by $e^{|v|}$, up to a constant, we obtain

$$
\left|\cos \left(t\langle\lambda, \lambda\rangle^{1 / 2}\right)\right|\left|\frac{\sin \left(t\langle\lambda, \lambda\rangle^{1 / 2}\right)}{\langle\lambda, \lambda\rangle^{1 / 2}}\right| \leq c e^{|t||v|} .
$$

We claim that $|v| \leq\|\operatorname{Im}(\lambda)\|$. One can see this as follows: since $\langle\lambda, \lambda\rangle=(u+i v)^{2}$, we have $u^{2}-v^{2}=\|\operatorname{Re}(\lambda)\|^{2}-\|\operatorname{Im}(\lambda)\|^{2}$ and $u v=\langle\operatorname{Re}(\lambda), \operatorname{Im}(\lambda)\rangle$. Thus, by Cauchy-Schwartz-Buniakowsly inequality, we have $u^{2} v^{2} \leq\|\operatorname{Re}(\lambda)\|^{2}\|\operatorname{Im}(\lambda)\|^{2}$, which is equivalent to $v^{2}\left(v^{2}+\|\operatorname{Re}(\lambda)\|^{2}-\|\operatorname{Im}(\lambda)\|^{2}\right) \leq\|\operatorname{Re}(\lambda)\|^{2}\|\operatorname{Im}(\lambda)\|^{2}$. This amounts to

$$
\left(v^{2}+\frac{\|\operatorname{Re}(\lambda)\|^{2}-\|\operatorname{Im}(\lambda)\|^{2}}{2}\right)^{2} \leq\left(\frac{\|\operatorname{Re}(\lambda)\|^{2}+\|\operatorname{Im}(\lambda)\|^{2}}{2}\right)^{2},
$$

which yields $v^{2} \leq\|\operatorname{Im}(\lambda)\|^{2}$, and the claim above holds. (We believe that the claim above is a well known fact. Now, by means of the Paley-Wiener Theorem 2 one can conclude that for all $N \geq 1$ and for every $k \in \mathscr{K}^{+}, u_{k}(a, t)$ is supported in the set

$$
\{(a, t) \in A \times \mathbb{R}|\|\log a\| \leq R+| t \mid\} .
$$

As an immediate consequence of Theorem 4 and in the light of the above observation, we obtain below our main result.

Theorem 5. (Huygens' Principle) The solution $u_{k}$ is supported in the shell

$$
\mathscr{C}=\{(a, t) \in A \times \mathbb{R}|| t|-R \leq\|\log a\| \leq| t \mid+R\}
$$

if and only if the root system $\mathscr{R}$ is reduced, $k_{\alpha} \in \mathbb{N}$ for all $\alpha \in \mathscr{R}$, and $N$ is odd starting from 3 . The set $\mathscr{C}$ is the union

$$
\bigcup_{\|\log b\| \leq R} \mathscr{C}_{b}
$$

where $\mathscr{C}_{b}$ is the light cone

$$
\mathscr{C}_{b}=\{(a, t) \in A \times \mathbb{R}|\|\log a-\log b\|=| t \mid\} .
$$

For the remainder of this section, we will restrict ourselves to the $W$-invariant situation. For $\lambda \in \mathfrak{a}_{\mathbb{C}}^{*}, k \in \mathscr{K}^{+}$, and $a \in A$, denote by $F(\lambda, k, a)$ the HeckmanOpdam hypergeometric function, which is, by construction, an eigenfunction for the hypergeometric system of differential equations. In particular

$$
\begin{aligned}
& \Delta(k)^{W} F(\lambda, k, a)=\langle\lambda, \lambda\rangle F(\lambda, k, a), \\
& F(\lambda, k, \mathbf{e})=1,
\end{aligned}
$$


where $\Delta(k)^{W}$ is the $W$-invariant part of the Dunkl-Cherednik Laplacian $\Delta(k)$, i.e.

$$
\Delta(k)^{W}=\sum_{j=1}^{N} \partial\left(\xi_{j}\right)^{2}+\sum_{\alpha \in \mathscr{R}^{+}} k_{\alpha} \operatorname{coth}(\alpha / 2) \partial_{\alpha}+\langle\rho(k), \rho(k)\rangle .
$$

Furthermore, in terms of the kernel $\mathbb{G}(\lambda, k, a)$ introduced in Theorem 1, we have

$$
\begin{aligned}
F(\lambda, k, a) & =\frac{1}{|W|} \sum_{w \in W} \mathbb{G}(\lambda, k, w a) \\
& =\frac{1}{|W|} \sum_{w \in W} \prod_{\alpha \in \mathscr{R}_{+}^{0}}\left(1-\frac{k_{\alpha}^{0}}{w \lambda(\breve{\alpha})}\right) \mathbb{G}(w \lambda, k, a) .
\end{aligned}
$$

We refer to $[16,17,23,24,18]$ for further details on Heckman-Opdam's theory of hypergeometric functions. Moreover, if $f \in \mathscr{C}_{c}^{\infty}(A)$ is a $W$-invariant function, then we may rewrite the Cherednik transform of $f$ as

$$
\mathscr{F}_{k}(f)(\lambda)=\int_{A} f(a) F(-i \lambda, k, a) d \mu(a)
$$

where $d \mu(a)$ is given by $(2.2)$. When the parameter $k$ corresponds to the root multiplicities of a Riemannian symmetric space of the noncompact type, the transform $\mathscr{F}_{k}$ coincides with the spherical Harish-Chandra transform [11]. The $W$-invariance of $f$ implies the $W$-invariance of $\mathscr{F}_{k}(f)$. Thus, the inverse transform of $\mathscr{F}_{k}(f)$ is given by

$$
f(a)=|W| \int_{\mathfrak{a}^{*}} \mathscr{F}_{k}(f)(\lambda) F(i \lambda, k, a) d \nu(\lambda),
$$

where $d \nu(\lambda)$ is given by $(2.5)$.

In this setting, the Cauchy problem (3.1) becomes

$$
\Delta(k)^{W} u_{k}^{W}(a, t)=\partial_{t t} u_{k}^{W}(a, t), \quad u_{k}^{W}(a, 0)=f^{W}(a), \partial_{t} u_{k}^{W}(a, 0)=g^{W}(a),
$$

where $f^{W}$ and $g^{W}$ are two $W$-invariant elements in $\mathscr{C}_{R}^{\infty}(A)$. Clearly

$$
\begin{aligned}
& u_{k}^{W}(a, t)=\sum_{w \in W} u_{k}(w a, t) \\
& =|W| \int_{\mathfrak{a}^{*}}\left\{\mathscr{F}_{k}\left(f^{W}\right)(\lambda) \cos (t\|\lambda\|)+\mathscr{F}_{k}\left(g^{W}\right)(\lambda) \frac{\sin (t\|\lambda\|)}{\|\lambda\|}\right\} F(i \lambda, k, a) d \nu(\lambda),
\end{aligned}
$$

where $u_{k}$ is the solution to the Cauchy problem (3.1). As an immediate consequence of Theorem 5 we have:

Theorem 6. Let $\mathscr{R}$ be a reduced root system with integral-valued multiplicity functions, and let $N$ be odd starting from 3 . Then the support of the solution $u_{k}^{W}$ to (3.6) is contained in the shell $\Gamma=\{(a, t) \in A \times \mathbb{R}|R-| t|\leq\|\log a\| \leq R+| t \mid\}$.

In these circumstances, and if in particular $k$ corresponds to the root multiplicities of a Riemannian symmetric space $G / K$ of the noncompact type, the above theorem implies the validity of Huygens' principle for the wave equation 
on $G / K$. The latter is a well known fact due to Solomatina [32], Helgason [14], Branson, Ólafsson, and Schlichtkrull [3], and to Chalykh and Veselov [5]. More precisely, let $G$ be a connected noncompact semisimple Lie group with finite center, and let $K$ be a maximal compact subgroup of $G$. The quotient manifold $G / K$ can be endowed with the structure of a Riemannian symmetric space of the noncompact type. Let $\theta$ be a Cartan involution on $G$ corresponding to $K$, i.e. $K=\{k \in G \mid \theta(k)=k\}$. Denote by the same letter the derived involution $\theta$ on $\mathfrak{g}:=\operatorname{Lie}(G)$. Let $\mathfrak{g}=\mathfrak{k} \oplus \mathfrak{p}$ be the Cartan decomposition of $\mathfrak{g}$ with respect to $\theta$. Assume that $\mathfrak{a}$ is a maximal abelian subspace of $\mathfrak{p}$, and let $\Sigma=\Sigma(\mathfrak{g}, \mathfrak{a})$ be the restricted root system of $\mathfrak{a}$ in $\mathfrak{g}$. For $\alpha \in \Sigma$, set $\mathfrak{g}_{\alpha}:=\{X \in \mathfrak{g} \mid[H, X]=\alpha(H) X$ for all $H \in \mathfrak{a}\}$ to be the corresponding root space, and let $m_{\alpha}:=\operatorname{dim}\left(\mathfrak{g}_{\alpha}\right)$. Now if we put $\mathscr{R}:=\{2 \alpha \mid \alpha \in \Sigma\}$ and $k_{2 \alpha}:=m_{\alpha} / 2$, for $\alpha \in \Sigma$, then $\Delta(k)^{W}$ coincides with the radial part of the Laplace-Beltrami operator on the symmetric space $G / K$, up to the term $\langle\rho(k), \rho(k)\rangle$ (cf. [13]). In this setting, the condition $\mathscr{R}$ is reduced with $k_{2 \alpha} \in \mathbb{N}$ amounts to the property that all Cartan subalgebras in $\mathfrak{g}$ are conjugate under the adjoint group of $\mathfrak{g}$. For completeness, we present the list of all Riemannian symmetric pairs $(\mathfrak{g}, \mathfrak{k})$ which satisfy the latter property. We refer to [13] for more details on Riemannian symmetric spaces.

Riemannian symmetric pairs with even multiplicity

\begin{tabular}{llccc}
\hline \multicolumn{1}{c}{$\mathfrak{g}$} & \multicolumn{1}{c}{$\mathfrak{k}$} & \multicolumn{1}{c}{$m_{\alpha}$} & Comments \\
\hline $\mathfrak{s l}(n, \mathbb{C})$ & $\mathfrak{s u}(n)$ & $A_{n-1}$ & 2 & $n \geq 2$ \\
$\mathfrak{s o}(2 n+1, \mathbb{C})$ & $\mathfrak{s o}(2 n+1)$ & $B_{n}$ & 2 & $n \geq 2$ \\
$\mathfrak{s p}(n, \mathbb{C})$ & $\mathfrak{s p}(n)$ & $C_{n}$ & 2 & $n \geq 3$ \\
$\mathfrak{s o}(2 n, \mathbb{C})$ & $\mathfrak{s o}(2 n)$ & $D_{n}$ & 2 & $n \geq 4$ \\
$\mathfrak{s o}(2 n+1,1)$ & $\mathfrak{s o}(2 n+1)$ & $A_{1}$ & $2 n$ & $n \geq 3$ \\
$\mathfrak{s} \mathfrak{u}^{*}(2 n)$ & $\mathfrak{s p}(n)$ & $A_{n-1}$ & 4 & $n \geq 2$ \\
$\left(\mathfrak{e}_{6}\right)_{\mathbb{C}}$ & $\mathfrak{e}_{6}$ & $E_{6}$ & 2 & - \\
$\left(\mathfrak{e}_{7}\right)_{\mathbb{C}}$ & $\mathfrak{e}_{7}$ & $E_{7}$ & 2 & - \\
$\left(\mathfrak{e}_{8}\right)_{\mathbb{C}}$ & $\mathfrak{e}_{8}$ & $E_{8}$ & 2 & - \\
$\left(\mathfrak{f}_{4}\right)_{\mathbb{C}}$ & $\mathfrak{f}_{4}$ & $F_{4}$ & 2 & - \\
$\left(\mathfrak{g}_{2}\right)_{\mathbb{C}}$ & $\mathfrak{g}_{2}$ & $G_{2}$ & 2 & - \\
$\mathfrak{e}_{6}(-26)$ & $\mathfrak{f}_{4(-20)}$ & $A_{2}$ & 8 & - \\
\hline
\end{tabular}




\section{Special isomorphisms of Riemannian} symmetric pairs with even multiplicity

\begin{tabular}{ccc}
\hline $\mathfrak{g}$ & $\mathfrak{k}$ \\
\hline $\mathfrak{s p}(1, \mathbb{C}) \approx \mathfrak{s l}(2, \mathbb{C})$ & $\mathfrak{s p}(1) \approx \mathfrak{s u}(2)$ \\
$\mathfrak{s o}(3, \mathbb{C}) \approx \mathfrak{s l}(2, \mathbb{C})$ & $\mathfrak{s o}(3) \approx \mathfrak{s u}(2)$ \\
$\mathfrak{s p}(2, \mathbb{C}) \approx \mathfrak{s o}(5, \mathbb{C})$ & $\mathfrak{s p}(2) \approx \mathfrak{s o}(5)$ \\
$\mathfrak{s o}(6, \mathbb{C}) \approx \mathfrak{s l}(4, \mathbb{C})$ & $\mathfrak{s o}(6) \approx \mathfrak{s u}(4)$ \\
$\mathfrak{s o}(3,1) \approx \mathfrak{s l}(2, \mathbb{C})$ & $\mathfrak{s o}(3) \approx \mathfrak{s u}(2)$ \\
$\mathfrak{s o}(5,1) \approx \mathfrak{s u}(4)$ & $\mathfrak{s o}(5) \approx \mathfrak{s p}(2)$ \\
\hline
\end{tabular}

Finally, we will give explicit expressions for the solution $u_{k}^{W}$ to (3.6). These formulas are obtained by means of the well known expressions of the solution to the classical Euclidean wave equation. This approach was used earlier in [4] for the solution to the wave equation associated with compact Riemannian symmetric spaces.

In [28, Theorem 5.1], the authors were able to give an explicit formula for the Heckman-Opdam hypergeometric functions, when $\mathscr{R}$ is reduced and $k_{\alpha} \in \mathbb{N}$ for all $\alpha \in \mathscr{R}$. More precisely, if

$$
\Delta(k, a):=\prod_{\alpha \in \mathscr{R}^{+}}\left(e^{\alpha(\log a)}-e^{-\alpha(\log a)}\right)^{k_{\alpha}}, \quad a \in A,
$$

then, for a reduced root system $\mathscr{R}$ with $k_{\alpha} \in \mathbb{N}$ for all $\alpha \in \mathscr{R}$, there exists a $W$-invariant differential operator $\mathbb{D}(k) \in \mathbb{C}\left[A_{\mathbb{C}}\right] \otimes \mathbb{S}\left(\mathfrak{a}_{\mathbb{C}}\right)$ such that

$$
F(\lambda, k, a)=\Delta(-k, a) \prod_{\alpha \in \mathscr{R}^{+}} \prod_{m=0}^{k_{\alpha}-1}\left(m^{2}-\lambda(\breve{\alpha})^{2}\right)^{-1} \mathbb{D}(k)\left(\sum_{w \in W} e^{w \lambda(X)}\right),
$$

where $a=\exp (X) \in A$. In [28, Theorem 4.10] the operator $\mathbb{D}(k)$ is expressed in terms of the Opdam shift operators. Using the $W$-invariance of the functions

$$
\lambda \mapsto \mathscr{F}_{k}\left(f^{W}\right)(\lambda), \quad \lambda \mapsto \mathscr{F}_{k}\left(g^{W}\right)(\lambda), \quad \lambda \mapsto \cos (t\|\lambda\|), \quad \lambda \mapsto \sin (t\|\lambda\|) /\|\lambda\|,
$$

we obtain

$$
\begin{aligned}
u_{k}^{W}(a, t) & =(2 \pi)^{-N}|W| \widetilde{c}(\rho(k), k)^{2} \Delta(-k, a) \times \\
& \mathbb{D}(k)\left[\int_{\mathfrak{a}^{*}}\left\{\mathscr{F}_{k}\left(f^{W}\right)(\lambda) \cos (t\|\lambda\|)+\mathscr{F}_{k}\left(g^{W}\right)(\lambda) \frac{\sin (t\|\lambda\|)}{\|\lambda\|}\right\} e^{i \lambda(X)} d \lambda\right],
\end{aligned}
$$

where $a=\exp (X) \in A$.

On the other hand, if $\Delta_{\mathfrak{a}}$ denotes the usual Laplacian operator on $\mathfrak{a} \cong \mathbb{R}^{N}$, and if we denote the Euclidean Fourier transform of a function $\psi$ on $\mathfrak{a}$ by $\widehat{\psi}$, 
then, for $X \in \mathfrak{a} \cong \mathbb{R}^{N}$, the function

$$
v(X, t):=\int_{\mathfrak{a}^{*}}\left\{\mathscr{F}_{k}\left(f^{W}\right)(\lambda) \cos (t\|\lambda\|)+\mathscr{F}_{k}\left(g^{W}\right)(\lambda) \frac{\sin (t\|\lambda\|)}{\|\lambda\|}\right\} e^{i \lambda(X)} d \lambda
$$

satisfies

$$
\Delta_{\mathfrak{a}} v(X, t)=\partial_{t t} v(X, t), \quad v(X, 0)=\psi(X), \quad \partial_{t} v(X, 0)=\phi(X),
$$

where $\psi, \phi \in \mathscr{C}^{\infty}(\mathfrak{a})^{W}$ such that $\widehat{\psi}=\mathscr{F}_{k}\left(f^{W}\right)$ and $\widehat{\phi}=\mathscr{F}_{k}\left(g^{W}\right)$.

For $r>0$, let $S_{r}(X) \subset \mathfrak{a}$ be the Euclidean sphere of radius $r$ and center $X$, and let $\Omega_{N}(r)$ be the surface area of $S_{r}(X)$. For a complex-valued function $f$ on $\mathfrak{a}$, set

$$
M^{r}(f)(X)=\frac{1}{\Omega_{N}(r)} \int_{S_{r}(X)} f(Y) d \sigma(Y)
$$

to be the mean value of $f$ on $S_{r}(X)$, where $d \sigma$ is the $O(N)$-invariant measure on $S_{r}(X)$. The following two facts can be found in [15, p. 481]:

(i) Put

$$
I_{r}(f)(X)=\left(\frac{\partial}{\partial\left(r^{2}\right)}\right)^{(N-3) / 2}\left(r^{N-2} M^{r}(f)(X)\right) .
$$

If $N \geq 2$ is odd, then the solution to (3.8) is given by

$$
v(X, t)=\frac{\Omega_{N}(1)}{(N-3) ! \Omega_{N-1}(1)}\left[\frac{\partial}{\partial t}\left(I_{t}(\psi)(X)\right)+I_{t}(\phi)(X)\right], \quad(t>0),
$$

where $\Omega_{N}(1)=2 \pi^{N / 2} \Gamma\left(\frac{N}{2}\right)^{-1}$.

(ii) Put

$$
J_{r}(f)(X)=\left(\frac{\partial}{\partial\left(r^{2}\right)}\right)^{(N-2) / 2}\left(r^{N-2} M^{r}(f)(X)\right) .
$$

If $N \geq 2$ is even, then, for $t>0$, the solution to (3.8) is given by

$$
\begin{aligned}
& v(X, t)= \\
& \frac{1}{(N-2) !}\left[\frac{\partial}{\partial t} \int_{0}^{t} r\left(t^{2}-r^{2}\right)^{-1 / 2} J_{r}(\psi)(X) d r+\int_{0}^{t} r\left(t^{2}-r^{2}\right)^{-1 / 2} J_{r}(\phi)(X) d r\right] .
\end{aligned}
$$

As an immediate consequence of (3.7) and the above two facts, we obtain the following explicit expressions for the solution $u_{k}^{W}(a, t)$.

Corollary 7. Let $\mathscr{R}$ be a reduced root system such that $k_{\alpha} \in \mathbb{N}$ for all $\alpha \in \mathscr{R}$, and assume that $N \geq 2$. There exists a differential operator $\mathbb{D}(k) \in \mathbb{C}\left[A_{\mathbb{C}}\right] \otimes \mathbb{S}\left(\mathfrak{a}_{\mathbb{C}}\right)$ such that, for all $t>0$, the solution $u_{k}^{W}(a, t)$ to the Cauchy problem (3.6) is given by:

(i) If $N$ is odd

$$
\begin{aligned}
& u_{k}^{W}(a, t)= \frac{(2 \pi)^{-N}|W| \Omega_{N}(1)}{(N-3) ! \Omega_{N-1}(1)} \widetilde{c}(\rho(k), k)^{2} \Delta(-k, a) \times \\
& \mathbb{D}(k)\left[\frac{\partial}{\partial t}\left(I_{t}\left(\mathscr{F}_{k}\left(f^{W}\right)^{\vee}\right)(X)\right)+I_{t}\left(\mathscr{F}_{k}\left(g^{W}\right)^{\vee}\right)(X)\right],
\end{aligned}
$$


where $a=\exp (X) \in A$. Here $\vee$ denotes the inverse of the Euclidean Fourier transform.

(ii) If $N$ is even

$$
\begin{aligned}
& u_{k}^{W}(a, t)=\frac{(2 \pi)^{-N}|W|}{(N-2) !} \widetilde{c}(\rho(k), k)^{2} \Delta(-k, a) \times \\
& \mathbb{D}(k)\left[\frac{\partial}{\partial t} \int_{0}^{t} r\left(t^{2}-r^{2}\right)^{-1 / 2} J_{r}\left(\mathscr{F}_{k}\left(f^{W}\right)^{\vee}\right)(X) d r\right. \\
&\left.\quad+\int_{0}^{t} r\left(t^{2}-r^{2}\right)^{-1 / 2} J_{r}\left(\mathscr{F}_{k}\left(g^{W}\right)^{\vee}\right)(X) d r\right],
\end{aligned}
$$

where $a=\exp (X) \in A$.

\section{References}

[1] S. Ben Said and B. Ørsted, The wave equation for the Dunkl operators. Preprint 2004.

[2] Y. Berest and A.P. Veselov, Huygens' principle and integrability, Russian Math. Surveys 49 (1994), 5-77.

[3] T. Branson, G. Ólafsson, and H. Schlichtkrull, Huygens' Principle in Riemannian Symmetric Spaces Math. Ann. 301 (1995), 445-462.

[4] T. Branson, G. Ólafsson, and A. Pasquale, The Paley-Wiener theorem and the local Huygens' principle for compact symmetric spaces. Preprint 2004.

[5] O.A. Chalykh and A.P. Veselov, Integrability and Huygens' principle on the symmetric spaces, Comm. Math. Phys. 178 (1996), 311-338.

[6] O.A. Chalykh, M.V. Feigin, and A.P. Veselov, New integrable generalizations of the Calogero-Moser quantum problem, J. Math. Phys. 39 (1998), 695-703.

[7] I. Cherednik, A unification of Knizhnik-Zamolodchikov equations and Dunkl operators via affine Hecke algebras, Invent. Math. 106 (1991), 411-432.

[8] R. Courant and D. Hilbert, Methods of mathematical physics, Vo. II, Interscience Publ., New York (1962).

[9] F.G. Friedlander, The wave equation on a curved space-time, Cambridge University Press, Cambridge-New York-Melbourne (1975).

[10] P. Günther, Huygens' principle and hyperbolic equations, Academic Press, Boston (1988)

[11] Harish-Chandra, Spherical functions on a semisimple Lie groups. I and II, Amer. J. Math. 80 (1958), 241-310, 553-613.

[12] J. Hadamard, Lectures on Cauchy's problem in linear partial differential equations, Yale University Press, New Haven (1923).

[13] S. Helgason, Differential geometry, Lie groups, and symmetric spaces, Academic Press, Inc., New York-London (1978).

[14]__ Huygens' principle for wave equations on symmetric spaces, J. Funct. Anal. 107 (1992), 279-288.

[15] Geometric analysis on symmetric spaces, Mathematical Surveys and Monographs 39, Amer. Math. Soc., Providense, RI (1994).

[16] G.J. Heckman, Root systems and hypergeometric functions. II, Compositio Math. 64 (1987), 353-373.

[17] G.J. Heckman and E.M. Opdam, Root systems and hypergeometric functions. I, Compositio Math. 64 (1987), 329-352.

[18] G.J. Heckman and H. Schlichtkrull, Harmonic analysis and special functions on symmetric spaces, Academic Press (1994).

[19] C. Huygens, Traité de la lumière, Van de Aa, Leyden (1690). English translation: Treatise on light, 1690, reprint by Dover Publications, New York (1962). 
[20] J.E. Lagnese and K.L. Stellmacher, A method of generating classes of Huygens' operators, J. Math. Mech. 17 (1967), 461-472.

[21] P.D. Lax and R.S. Phillips, Scattering theory, Academic Press, New York and London (1967).

[22] R.G. McLenaghan, Huygens' principle, Ann. Inst. Henri Poincaré 37 (1982), 211-236.

[23] E.M. Opdam, Root systems and hypergeometric functions. III, Compositio Math. 67 (1988), 21-49.

[24] $\_$Root systems and hypergeometric functions. IV, Compositio Math. 67 (1988), 191-209.

[25] _ Harmonic analysis for certain representations of graded Hecke algebras, Acta. Math. 175 (1995), 75-121.

[26] Lecture notes on Dunkl operators for real and complex reflection groups, AMS Memoire 8 (2000).

[27] G. Ólafsson, and H. Schlichtkrull, Wave propagation on Riemannian Symmetric Spaces J. Funct. Anal. 107 (1992), 270-278.

[28] G. Ólafsson and A. Pasquale, A Paley-Wiener theorem for the $\Theta$-hypergeometric transform: the even multiplicity case, J. Math. Pures Appl. 83 (2004), 869-927.

[29] B. Ørsted, The conformal invariance of Huygens' principle, J. Diff. Geom. 16 (1981), 1-9.

[30] K. Stellmacher, Ein Beispiel einer Huygensschen Differentialgleichung, Nachr. Akad. Wiss. Göttingen, Math.-Phys. Kl, IIa, Bd. 10 (1953), 133-138.

[31] R. Schimming, A review on Huygens' principle for linear hyperbolic differential operators, in: L.V. Ovsiannikov and N.H. Ibragimov (eds), Proceedings of the International Symposium Group-Theoretical Methods in Mechanics, Novosibirsk (1978).

[32] L.E. Solomatinia, Translation representation and Huygens' principle for an invariant wave equation in a Riemannian symmetric spaces, Sov. Math. Izv. 30 (1986), 108-111.

University of Aarhus, Department of Mathematical Sciences, Building 530, Ny Munkegade, DK-8000 Aarhus C, Denmark

E-mail address: ssaid@imf .au.dk 